\title{
Positive Solutions of a Singular Nonlocal Fractional Order Differential System via Schauder's Fixed Point Theorem
}

\author{
Xinguang Zhang, ${ }^{1,2}$ Cuiling Mao, ${ }^{1}$ Yonghong $\mathrm{Wu}^{2}$ and $\mathrm{Hua} \mathrm{Su}^{3}$ \\ ${ }^{1}$ School of Mathematical and Informational Sciences, Yantai University, Yantai, Shandong 264005, China \\ ${ }^{2}$ Department of Mathematics and Statistics, Curtin University of Technology, Perth, WA 6845, Australia \\ ${ }^{3}$ School of Mathematics and Quantitative Economics, Shandong University of Finance and Economics, \\ Jinan, Shandong 250014, China
}

Correspondence should be addressed to Xinguang Zhang; zxg123242@sohu.com

Received 24 March 2014; Revised 12 May 2014; Accepted 12 May 2014; Published 26 May 2014

Academic Editor: Xinan Hao

Copyright (C) 2014 Xinguang Zhang et al. This is an open access article distributed under the Creative Commons Attribution License, which permits unrestricted use, distribution, and reproduction in any medium, provided the original work is properly cited.

We establish the existence of positive solutions to a class of singular nonlocal fractional order differential system depending on two parameters. Our methods are based on Schauder's fixed point theorem.

\section{Introduction}

Differential equations of fractional order have recently proved to be valuable tools in the modeling of many phenomena in various fields of science and engineering. Particularly, fractional derivatives provide an excellent tool for the description of memory and hereditary properties of many materials and processes. With this advantage, fractional order models are more realistic and practical than the classical integer-order models in physics, biology, economics, control theory, signal and image processing, biophysics, blood flow phenomena, fitting of experimental data, and so forth [1-16]. Recently, Rehman and Khan [17] studied the fractional order multipoint boundary value problem:

$$
\begin{aligned}
& D_{t}^{\alpha} y(t)=f\left(t, y(t), D_{t}^{\beta} y(t)\right), \quad t \in(0,1), \\
& y(0)=0, \quad D_{t}^{\beta} y(1)-\sum_{i=1}^{m-2} \zeta_{i} D_{t}^{\beta} y\left(\xi_{i}\right)=y_{0},
\end{aligned}
$$

where $1<\alpha \leq 2,0<\beta<1, \zeta_{i} \in[0,+\infty), 0<$ $\xi_{i}<1$, with $\sum_{i=1}^{m-2} \zeta_{i} \xi_{i}<1$. By using the contraction mapping principle, the existence and uniqueness of positive solutions were established. In [18], Zhang et al. discussed the existence and uniqueness of positive solutions for the following fractional differential equation with derivatives:

$$
\begin{aligned}
-\mathscr{D}_{\mathbf{t}}^{\alpha} x(t) & =f\left(t, x(t),-\mathscr{D}_{\mathbf{t}}^{\beta} x(t)\right), \quad t \in(0,1), \\
\mathscr{D}_{\mathbf{t}}^{\beta} x(0) & =0, \quad \mathscr{D}_{\mathbf{t}}^{\gamma} x(1)=\sum_{j=1}^{p-2} a_{j} \mathscr{D}_{\mathbf{t}}^{\gamma} x\left(\xi_{j}\right),
\end{aligned}
$$

where $1<\alpha \leq 2, \alpha-\beta>1,0<\beta \leq \gamma<1,0<\xi_{1}<\xi_{2}<$ $\cdots<\xi_{p-2}<1, a_{j} \in[0,+\infty)$ with $c=\sum_{j=1}^{p-2} a_{j} \xi_{j}^{\alpha-\gamma-1}<1$, and $\mathscr{D}_{\mathrm{t}}$ is the standard Riemann-Liouville derivative. $f$ : $(0,1) \times[0,+\infty) \times(-\infty,+\infty) \rightarrow[0,+\infty)$ is continuous, and $f(t, u, v)$ may be singular at $t=0,1$. By means of monotone iterative technique, the existence and uniqueness of the positive solution for a fractional differential equation with derivatives are established, and the iterative sequence of the solution, an error estimation, and the convergence rate of the positive solution are also given.

However, the research on the systems of fractional differential equations has not received much attention. So motivated by the results mentioned above, in this paper, we study the existence of positive solutions for the following singular 
nonlocal fractional order differential system depending on two parameters:

$$
\begin{gathered}
-D_{t}^{\alpha} u(t)=\lambda f\left(t, D_{t}^{\beta_{1}} v(t)\right), \\
-D_{t}^{\beta} v(t)=\mu g\left(t, D_{t}^{\alpha_{1}} u(t)\right), \\
0<t<1, \\
D_{t}^{\alpha_{1}} u(0)=0, \quad D_{t}^{\gamma_{1}} u(1)=\sum_{j=1}^{m-2} b_{j} D_{t}^{\gamma_{1}} u\left(\zeta_{j}\right), \\
D_{t}^{\beta_{1}} v(0)=0, \quad D_{t}^{\delta_{1}} v(1)=\sum_{j=1}^{m-2} a_{j} D_{t}^{\delta_{1}} v\left(\xi_{j}\right),
\end{gathered}
$$

where $1<\alpha, \beta \leq 2, \alpha-\gamma_{1} \geq 1, \beta-\delta_{1} \geq 1,0 \leq \beta_{1} \leq \delta_{1}$, $0 \leq \alpha_{1} \leq \gamma_{1}, a_{j}, b_{j} \in[0,+\infty), \xi_{i}, \zeta_{i} \in(0,1)$ with $0<$ $\sum_{j=1}^{m-2} a_{j} \xi_{j}, \sum_{j=1}^{m-2} b_{j} \zeta_{j}<1, D_{t}^{\alpha}, D_{t}^{\beta}$ are the standard RiemannLiouville derivatives, and $\lambda$ and $\mu$ are positive parameters. $f:(0,1) \times(0,+\infty) \rightarrow[0,+\infty)$ and $g:[0,1] \times[0,+\infty) \rightarrow$ $(0,+\infty)$ are continuous and $f(t, x)$ may be singular at $t=0,1$ and $x=0$. The system (3) is an abstract model arising from biological dynamic system, which was introduced by Perelson [5] to describe the primary infection with HIV in integerorder version, and was extended to a fractional order version of HIV-1 infection of CD4 ${ }^{+}$T-cells by Arafa et al. [11].

The present paper has several interesting features. Firstly, the system depends on two parameters and the nonlinear terms $f$ and $g$ are allowed to have different nonlinear character; that is, $f$ is decreasing on $y$ and $g$ is increasing on $x$; secondly, $f$ may be singular at $y=0$ and $t=0,1$; so far fewer work was done when $f$ can be singular at $y=0$; thirdly, the boundary conditions of the system are nonlocal and involve fractional derivatives of the unknown functions.

\section{Preliminaries and Lemmas}

In this section, we firstly define an appropriate invariant set and then make a change of variables for the system (3) so that Schauder's fixed point theorem can be applied. Our work is based on fractional framework; for further background knowledge of fractional calculus, we refer readers to the monographs [1-4] or the papers $[6,8,17,18]$ and the references therein.

Throughout this paper, we mean by $C[0,1]$ the Banach space of all continuous functions on $[0,1]$ with the usual norm $\|x\|=\max _{0 \leq t \leq 1}|x(t)|$. Let

$$
P=\{x \in C[0,1]: x(t) \geq 0, t \in[0,1]\}
$$

then $P$ is a normal cone in the Banach space $E$. Thus the space $C[0,1]$ can be equipped with a partial order given by

$x, y \in C[0,1], x-y \in P \Longleftrightarrow x(t) \geq y(t), \quad$ for $t \in[0,1]$.
Now define a subcone of $P$ as follows:

$$
\begin{aligned}
B=\{x(t) \in P: \text { there exist two } \\
\text { positive numbers } L_{x}>1>l_{x} \\
\left.\quad \text { such that } l_{x} t^{\alpha-\alpha_{1}-1} \leq x(t) \leq L_{x} t^{\alpha-\alpha_{1}-1}, t \in[0,1]\right\} .
\end{aligned}
$$

Obviously, $B$ is nonempty since $t^{\alpha-\alpha_{1}-1} \in B$.

Lemma 1. Let $u(t)=I^{\alpha_{1}} x(t), v(t)=I^{\beta_{1}} y(t), x(t)$, and $y(t) \epsilon$ $C[0,1]$. Then system (3) is turned into the equivalent one:

$$
\begin{gathered}
-D_{t}^{\alpha-\alpha_{1}} x(t)=\lambda f(t, y(t)), \\
-D_{t}^{\beta-\beta_{1}} y(t)=\mu g(t, x(t)), \\
0<t<1, \\
x(0)=0, \quad D_{t}^{\gamma_{1}-\alpha_{1}} x(1)=\sum_{j=1}^{m-2} b_{j} D_{t}^{\gamma_{1}-\alpha_{1}} x\left(\zeta_{j}\right), \\
y(0)=0, \quad D_{t}^{\delta_{1}-\beta_{1}} y(1)=\sum_{j=1}^{m-2} a_{j} D_{t}^{\delta_{1}-\beta_{1}} y\left(\xi_{j}\right),
\end{gathered}
$$

and if $(x, y) \in C[0,1] \times C[0,1]$ is a solution of the problem (7), then $\left(I^{\alpha_{1}} x(t), I^{\beta_{1}} y(t)\right)$ is a solution of the system (3).

Proof. By using semigroup property of the fractional integration operator (see [1] page 73, Lemma 2.3 or [4] Sections 2.3 and 2.5), one has

$$
\begin{aligned}
& D_{t}^{\alpha} u(t)=\frac{d^{n}}{d t^{n}}\left(I^{n-\alpha} I^{\alpha_{1}} x(t)\right) \\
&=\frac{d^{n}}{d t^{n}} I^{n-\alpha+\alpha_{1}} x(t)=D_{t}^{\alpha-\alpha_{1}} x(t), \\
& D_{t}^{\gamma_{1}} u(t)=\frac{d^{n}}{d t^{n}}\left(I^{n-\gamma_{1}} I^{\alpha_{1}} x(t)\right) \\
&=\frac{d^{n}}{d t^{n}} I^{n-\gamma_{1}+\alpha_{1}} x(t)=D_{t}^{\gamma_{1}-\alpha_{1}} x(t), \\
& D_{t}^{\alpha_{1}} u(t)=D_{t}^{\alpha_{1}} I^{\alpha_{1}} x(t)=x(t) .
\end{aligned}
$$

And then, it follows from (8) that

$$
\begin{aligned}
& D_{t}^{\alpha_{1}} u(0)=x(0)=0 \\
& D_{t}^{\gamma_{1}-\alpha_{1}} x(1)= D_{t}^{\gamma_{1}} u(1) \\
&= \sum_{j=1}^{m-2} b_{j} D_{t}^{\gamma_{1}} u\left(\zeta_{j}\right)=\sum_{j=1}^{m-2} b_{j} D_{t}^{\gamma_{1}-\alpha_{1}} x\left(\zeta_{j}\right) .
\end{aligned}
$$

In the same way, we also have

$$
y(0)=0, \quad D_{t}^{\delta_{1}-\beta_{1}} y(1)=\sum_{j=1}^{m-2} a_{j} D_{t}^{\delta_{1}-\beta_{1}} y\left(\xi_{j}\right) .
$$


It follows from $u(t)=I^{\alpha_{1}} x(t)$ and $v(t)=I^{\beta_{1}} y(t)$ that $x(t)=D_{t}^{\alpha_{1}} u(t)$ and $y(t)=D_{t}^{\beta_{1}} v(t)$. So substituting the above formulas into (3), we obtain (7).

On the other hand, if $(x, y)$ is a solution of (7), (8) yields

$$
\begin{aligned}
&-D_{t}^{\alpha} u(t)=-\frac{d^{n}}{d t^{n}} I^{n-\alpha} I^{\alpha_{1}} x(t)=-\frac{d^{n}}{d t^{n}} I^{n-\alpha-\alpha_{1}} x(t) \\
&=-D_{t}^{\alpha-\alpha_{1}} x(t)=\lambda f(t, y(t))=\lambda f\left(t, D_{t}^{\beta_{1}} v(t)\right), \\
&-D_{t}^{\beta} v(t)=-D_{t}^{\beta-\beta_{1}} y(t)=\mu g(t, x(t))=\mu g\left(t, D_{t}^{\alpha_{1}} u(t)\right), \\
& D_{t}^{\alpha_{1}} u(t)=D_{t}^{\alpha_{1}} I^{\alpha_{1}} x(t)=x(t), \quad D_{t}^{\gamma_{1}} u(t)=D_{t}^{\gamma_{1}-\alpha_{1}} x(t), \\
& D_{t}^{\beta_{1}} v(t)=D_{t}^{\beta_{1}} I^{\beta_{1}} y(t)=y(t), \quad D_{t}^{\delta_{1}} v(t)=D_{t}^{\delta_{1}-\beta_{1}} y(t) .
\end{aligned}
$$

So from (11), we have

$$
\begin{array}{ll}
D_{t}^{\alpha_{1}} u(0)=0, & D_{t}^{\gamma_{1}} u(1)=\sum_{j=1}^{m-2} b_{j} D_{t}^{\gamma_{1}} u\left(\zeta_{j}\right), \\
D_{t}^{\beta_{1}} v(0)=0, & D_{t}^{\delta_{1}} v(1) \sum_{j=1}^{m-2} a_{j} D_{t}^{\delta_{1}-\beta_{1}} v\left(\xi_{j}\right) .
\end{array}
$$

Moreover

$$
I^{\alpha_{1}} x(t), \quad I^{\beta_{1}} y(t) \in C[0,1] .
$$

Consequently, $\left(I^{\alpha_{1}} x(t), I^{\beta_{1}} y(t)\right)$ is a positive solution of (3).

Now we recall some useful lemmas by [18], which are important to the proof of our main results.

Lemma 2 (see [18]). Let $h \in L^{1}[0,1]$, if $1<\alpha-\alpha_{1} \leq 2$; then the unique solution of the linear problem

$$
\begin{gathered}
-D_{t}^{\alpha-\alpha_{1}} x(t)=h(t), \quad t \in(0,1), \\
x(0)=0, \quad D_{t}^{\gamma_{1}-\alpha_{1}} x(1)=\sum_{j=1}^{m-2} b_{j} D_{t}^{\gamma_{1}-\alpha_{1}} x\left(\zeta_{j}\right),
\end{gathered}
$$

is given by

$$
x(t)=\int_{0}^{1} K_{\alpha}(t, s) h(s) d s,
$$

where

$$
\begin{aligned}
& K_{\alpha}(t, s) \\
& \quad=K_{\alpha 1}(t, s)+\frac{t^{\alpha-\alpha_{1}-1}}{1-\sum_{j=1}^{m-2} b_{j} \zeta_{j}^{\alpha-\gamma_{1}-1}} \sum_{j=1}^{m-2} b_{j} K_{\alpha 2}\left(\zeta_{j}, s\right),
\end{aligned}
$$

$$
\begin{aligned}
& K_{\alpha 1}(t, s) \\
& =\frac{1}{\Gamma\left(\alpha-\alpha_{1}\right)}\left\{\begin{array}{r}
t^{\alpha-\alpha_{1}-1}(1-s)^{\alpha-\gamma_{1}-1}-(t-s)^{\alpha-\alpha_{1}-1}, \\
0 \leq s \leq t \leq 1, \\
t^{\alpha-\alpha_{1}-1}(1-s)^{\alpha-\gamma_{1}-1}, \quad \\
0 \leq t \leq s \leq 1,
\end{array}\right. \\
& \quad=\frac{1}{\Gamma\left(\alpha-\alpha_{1}\right)}\left\{\begin{array}{r}
(t(1-s))^{\alpha-\gamma_{1}-1}-(t-s)^{\alpha-\gamma_{1}-1}, \\
0 \leq s \leq t \leq 1, \\
(t(1-s))^{\alpha-\gamma_{1}-1}, \\
0 \leq t \leq s \leq 1 .
\end{array}\right.
\end{aligned}
$$

Moreover, for any $t, s \in[0,1]$,

$$
K_{\alpha i}(t, s) \leq \frac{(1-s)^{\alpha-\gamma_{1}-1}}{\Gamma\left(\alpha-\alpha_{1}\right)}, \quad i=1,2
$$

By Lemma 2, similar results are valid for the problem

$$
\begin{gathered}
-D_{t}^{\beta-\beta_{1}} y(t)=h(t), \quad t \in[0,1], \\
y(0)=0, \quad D_{t}^{\delta_{1}-\beta_{1}} y(1)=\sum_{j=i}^{m-2} a_{j} D_{t}^{\delta_{1}-\beta_{1}} y\left(\xi_{j}\right) .
\end{gathered}
$$

For convenience, we adopt the following corresponding notations:

$$
\begin{aligned}
& K_{\beta}(t, s) \\
& \quad=K_{\beta 1}(t, s)+\frac{t^{\beta-\beta_{1}-1}}{1-\sum_{j=1}^{m-2} a_{j} \xi_{j}^{\beta-\delta_{1}-1}} \sum_{j=1}^{m-2} a_{j} K_{\beta 2}\left(\xi_{j}, s\right),
\end{aligned}
$$

$K_{\beta 1}(t, s)$

$$
=\frac{1}{\Gamma\left(\beta-\beta_{1}\right)} \begin{cases}t^{\beta-\beta_{1}-1}(1-s)^{\beta-\delta_{1}-1}-(t-s)^{\beta-\beta_{1}-1}, \\ t^{\beta-\beta_{1}-1}(1-s)^{\beta-\delta_{1}-1}, & 0 \leq s \leq t \leq 1, \\ & 0 \leq t \leq s \leq 1,\end{cases}
$$

$$
K_{\beta 2}(t, s)
$$

$$
=\frac{1}{\Gamma\left(\beta-\beta_{1}\right)}\left\{\begin{array}{r}
(t(1-s))^{\beta-\delta_{1}-1}-(t-s)^{\beta-\delta_{1}-1}, \\
0 \leq s \leq t \leq 1, \\
(t(1-s))^{\beta-\delta_{1}-1}, \quad \\
0 \leq t \leq s \leq 1 .
\end{array}\right.
$$

Lemma 3 (see [18]). The Green functions $K_{\alpha}(t, s)$ and $K_{\beta}(t, s)$ have the following properties:

(1) $K_{\alpha}(t, s)>0$ and $K_{\beta}(t, s)>0$ for $t, s \in(0,1)$, 
(2) there exist functions $m_{\alpha}(s), m_{\beta}(s), M_{\alpha}(s)$, and $M_{\beta}(s)$ such that

$$
\begin{aligned}
& m_{\alpha}(s) t^{\alpha-\alpha_{1}-1} \leq K_{\alpha}(t, s) \leq M_{\alpha}(s) t^{\alpha-\alpha_{1}-1}, \quad t, s \in(0,1), \\
& m_{\beta}(s) t^{\beta-\beta_{1}-1} \leq K_{\beta}(t, s) \leq M_{\beta}(s) t^{\beta-\beta_{1}-1}, \quad t, s \in(0,1),
\end{aligned}
$$

where

$$
\begin{aligned}
& m_{\alpha}(s)=\frac{\sum_{j=1}^{m-2} b_{j} K_{\alpha 2}\left(\zeta_{j}, s\right)}{1-\sum_{j=1}^{m-2} b_{j} \zeta_{j}^{\alpha-\gamma_{1}-1}}, \\
& M_{\alpha}(s)=\frac{1}{\Gamma\left(\alpha-\alpha_{1}\right)}+\frac{\sum_{j=1}^{m-2} b_{j} K_{\alpha 2}\left(\zeta_{j}, s\right)}{1-\sum_{j=1}^{m-2} b_{j} \zeta_{j}^{\alpha-\gamma_{1}-1}}, \\
& m_{\beta}(s)=\frac{\sum_{j=1}^{m-2} a_{j} K_{\beta 2}\left(\xi_{j}, s\right)}{1-\sum_{j=1}^{m-2} a_{j} \xi_{j}^{\beta-\delta_{1}-1}}, \\
& M_{\beta}(s)=\frac{1}{\Gamma\left(\beta-\beta_{1}\right)}+\frac{\sum_{j=1}^{m-2} a_{j} K_{\beta 2}\left(\xi_{j}, s\right)}{1-\sum_{j=1}^{m-2} a_{j} \xi_{j}^{\beta-\delta_{1}-1}} .
\end{aligned}
$$

Clearly, the following maximum principle is direct conclusion of Lemma 2.

Lemma 4. If $1<\alpha-\alpha_{1} \leq 2$ and $x \in C([0,1], R)$ satisfies

$$
x(0)=0, \quad D_{t}^{\gamma_{1}-\alpha_{1}} x(1)=\sum_{j=1}^{m-2} b_{j} D_{t}^{\gamma_{1}-\alpha_{1}} x\left(\zeta_{j}\right),
$$

and $-D_{t}^{\alpha-\alpha_{1}} x(t) \geq 0$ for any $t \in(0,1)$, then $x(t) \geq 0$ for $t \in$ $(0,1)$.

It is well known that $(x, y) \in C(0,1) \times C[0,1]$ is a solution of the system (7) if and only if $(x, y)$ is a solution of the nonlinear integral system of equations

$$
\begin{aligned}
& x(t)=\lambda \int_{0}^{1} K_{\alpha}(t, s) f(s, y(s)) d s, \\
& y(t)=\mu \int_{0}^{1} K_{\beta}(t, s) g(s, x(s)) d s,
\end{aligned}
$$

and the system (23) is equivalent to the following nonlinear integral equation

$$
\begin{array}{r}
x(t)=\lambda \int_{0}^{1} K_{\alpha}(t, s) f\left(s, \mu \int_{0}^{1} K_{\beta}(s, \tau) g(\tau, x(\tau)) d \tau\right) d s \\
t \in[0,1] .
\end{array}
$$

Let us define a nonlinear operator $(F x)(t)$ by

$$
\begin{array}{r}
(F x)(t) \\
=\lambda \int_{0}^{1} K_{\alpha}(t, s) f\left(s, \mu \int_{0}^{1} K_{\beta}(s, \tau) g(\tau, x(\tau)) d \tau\right) d s, \\
t \in[0,1] .
\end{array}
$$

Then the existence of solutions to the system of (7) is equivalent to the existence of fixed point of the nonlinear operator $F$; that is, if $x^{*}(t)$ is a fixed point of $F$ in $C[0,1]$, then system (7) has at least one solution $\left(x^{*}(t), y^{*}(t)\right)$ which can be written by

$$
\begin{aligned}
x(t) & =x^{*}(t), \\
y^{*}(t) & =\mu \int_{0}^{1} K_{\beta}(t, s) g\left(s, x^{*}(s)\right) d s,
\end{aligned}
$$

and then system (3) has at least one solution:

$$
\left(I^{\alpha_{1}} x^{*}(t), I^{\beta_{1}} y^{*}(t)\right) .
$$

In order to find the fixed point of $F$, we need the definitions of the upper solution and lower solution for the following integrodifferential equation:

$$
\begin{gathered}
-D_{t}^{\alpha-\alpha_{1}} x(t)=\lambda f\left(t, \mu \int_{0}^{1} K_{\beta}(t, s) g(s, x(s)) d s\right), \\
x(0)=0, \quad D_{t}^{\gamma_{1}-\alpha_{1}} x(1)=\sum_{j=1}^{m-2} b_{j} D_{t}^{\gamma_{1}-\alpha_{1}} x\left(\zeta_{j}\right) .
\end{gathered}
$$

Definition 5. A continuous function $\phi(t)$ is called a lower solution of the problem (28), if it satisfies

$$
\begin{gathered}
-D_{t}^{\alpha-\alpha_{1}} \phi(t) \leq \lambda f\left(t, \mu \int_{0}^{1} K_{\beta}(t, s) g(s, \phi(s)) d s\right), \\
\phi(0) \geq 0, \quad D_{t}^{\gamma_{1}-\alpha_{1}} \phi(1) \geq \sum_{j=1}^{m-2} b_{j} D_{t}^{\gamma_{1}-\alpha_{1}} \phi\left(\zeta_{j}\right) .
\end{gathered}
$$

Definition 6. A continuous function $\varphi(t)$ is called an upper solution of the problem (28), if it satisfies

$$
\begin{gathered}
-D_{t}^{\alpha-\alpha_{1}} \varphi(t) \geq \lambda f\left(t, \mu \int_{0}^{1} K_{\beta}(t, s) g(s, \varphi(s)) d s\right), \\
\varphi(0) \leq 0, \quad D_{t}^{\gamma_{1}-\alpha_{1}} \varphi(1) \leq \sum_{j=1}^{m-2} b_{j} D_{t}^{\gamma_{1}-\alpha_{1}} \varphi\left(\zeta_{j}\right) .
\end{gathered}
$$

\section{Main Result}

For the convenience in presentation, we now present some assumptions to be used in the rest of the paper.

(A1) $f:(0,1) \times(0,+\infty) \rightarrow[0,+\infty)$ is continuous and decreasing on $y$ in $(0,+\infty)$ and $g:[0,1] \times[0,+\infty) \rightarrow$ $(0,+\infty)$ is continuous and increasing on $x$ in $[0,+\infty)$.

(A2) For any real numbers $l, \mu>0$,

$$
\int_{0}^{1} M_{\alpha}(s) f\left(s, \mu \int_{0}^{1} K_{\beta}(s, \tau) g\left(\tau, l \tau^{\alpha-\alpha_{1}-1}\right) d \tau\right) d s<+\infty .
$$

Theorem 7. Suppose (A1) and (A2) hold; then for any $(\lambda, \mu) \in$ $(0,+\infty) \times(0,+\infty)$, the system (3) has at least one positive 
solution $\left(u^{*}, v^{*}\right)$, and there exist positive constants $r_{1}^{*}, r_{2}^{*}, d_{1}^{*}$, and $d_{2}^{*}$ such that

$$
\begin{array}{r}
r_{1}^{*} t^{\alpha-1} \leq u^{*}(t) \leq r_{2}^{*} t^{\alpha-1} \\
d_{1}^{*} t^{\beta-1} \leq v^{*}(t) \leq d_{2}^{*} t^{\beta-1} \\
t \in[0,1]
\end{array}
$$

Proof. We start by showing that (3) has at least one positive solution $\left(u^{*}, v^{*}\right)$. For this purpose, we firstly prove that the operator $F$ is well defined and $F(B) \subset B$.

For any $x \in B$, there exist two positive numbers $l_{x}<1<$ $L_{x}$ such that $l_{x} t^{\alpha-\alpha_{1}-1} \leq x(t) \leq L_{x} t^{\alpha-\alpha_{1}-1}$, so it follows from (25), (20), and (31) that

$(F x)(t)$

$$
\begin{aligned}
& =\lambda \int_{0}^{1} K_{\alpha}(t, s) \\
& \quad \times f\left(s, \mu \int_{0}^{1} K_{\beta}(s, \tau) g(\tau, x(\tau)) d \tau\right) d s \\
& \leq \lambda t^{\alpha-\alpha_{1}-1} \\
& \quad \times \int_{0}^{1} M_{\alpha}(s) \\
& \quad \times f\left(s, \mu \int_{0}^{1} K_{\beta}(s, \tau) g\left(\tau, l_{x} \tau^{\alpha-\alpha_{1}-1}\right) d \tau\right) d s \\
& <+\infty,
\end{aligned}
$$

$(F x)(t)$

$$
\begin{aligned}
\geq & \lambda t^{\alpha-\alpha_{1}-1} \int_{0}^{1} m_{\alpha}(s) f\left(s, \mu \int_{0}^{1} K_{\beta}(s, \tau) g(\tau, x(\tau)) d \tau\right) d s \\
\geq & \lambda t^{\alpha-\alpha_{1}-1} \\
& \times \int_{0}^{1} m_{\alpha}(s) f\left(s, \mu \int_{0}^{1} K_{\beta}(s, \tau) g\left(\tau, L_{x} \tau^{\alpha-\alpha_{1}-1}\right) d \tau\right) d s .
\end{aligned}
$$

Take

$$
\begin{aligned}
l_{x}^{\prime} & \min \{1, \lambda \\
& \quad \times \int_{0}^{1} m_{\alpha}(s) \\
& \left.\quad \times f\left(s, \mu \int_{0}^{1} K_{\beta}(s, \tau) g\left(\tau, L_{x} \tau^{\alpha-\alpha_{1}-1}\right) d \tau\right) d s\right\},
\end{aligned}
$$

$$
\begin{aligned}
& L_{x}^{\prime} \\
& =\max \{1, \lambda \\
& \quad \times \int_{0}^{1} M_{\alpha}(s) \\
& \left.\quad \times f\left(s, \mu \int_{0}^{1} K_{\beta}(s, \tau) g\left(\tau, l_{x} \tau^{\alpha-\alpha_{1}-1}\right) d \tau\right) d s\right\} ;
\end{aligned}
$$

then

$$
l_{x}^{\prime} t^{\alpha-\alpha_{1}-1} \leq(F x)(t) \leq L_{x}^{\prime} t^{\alpha-\alpha_{1}-1} .
$$

So $(F x)(t)$ is well defined and $F(B) \subseteq B$. It follows from (A1) that the operator $(F x)(t)$ is decreasing on $x$. Moreover, by Lemma 2, we have

$$
\begin{aligned}
& -D_{t}^{\alpha-\alpha_{1}}(F x)(t)=\lambda f\left(t, \mu \int_{0}^{1} K_{\beta}(t, s) g(s, F x(s)) d s\right), \\
& (F x)(0)=0, \quad D_{t}^{\gamma_{1}-\alpha_{1}}(F x)(1)=\sum_{j=1}^{m-2} b_{j} D_{t}^{\gamma_{1}-\alpha_{1}} F x\left(\zeta_{j}\right) .
\end{aligned}
$$

Let

$$
\begin{aligned}
& \underline{e}(t)=\min \left\{t^{\alpha-\alpha_{1}-1}, F\left(t^{\alpha-\alpha_{1}-1}\right)\right\}, \\
& \bar{e}(t)=\max \left\{t^{\alpha-\alpha_{1}-1}, F\left(t^{\alpha-\alpha_{1}-1}\right)\right\} ;
\end{aligned}
$$

if $t^{\alpha-\alpha_{1}-1}=F\left(t^{\alpha-\alpha_{1}-1}\right)$, then

$$
x^{*}(t)=t^{\alpha-\alpha_{1}-1}, \quad y^{*}(t)=\mu \int_{0}^{1} K_{\beta}(t, s) g\left(s, s^{\alpha-\alpha_{1}-1}\right) d s,
$$

is positive solution of (7). Thus the system (3) has at least one solution

$$
\left(I^{\alpha_{1}} x^{*}(t), I^{\beta_{1}} y^{*}(t)\right)
$$

which satisfies Theorem 7. If $t^{\alpha-\alpha_{1}-1} \neq F\left(t^{\alpha-\alpha_{1}-1}\right)$, we have

$$
\underline{e}(t), \bar{e}(t) \in B, \quad \underline{e}(t) \leq t^{\alpha-\alpha_{1}-1} \leq \bar{e}(t) .
$$

Let

$$
\varphi(t)=(F \underline{e})(t), \quad \phi(t)=(F \bar{e})(t) .
$$

From (A1), we know that $F$ is nonincreasing on $x$; thus by (37) and (40), one gets

$$
\begin{gathered}
\phi(t)=(F \bar{e})(t) \leq F \underline{e}(t)=\varphi(t), \\
(F \bar{e})(t) \leq F\left(t^{\alpha-\alpha_{1}-1}\right) \leq \bar{e}(t), \\
(F \underline{e})(t) \geq F\left(t^{\alpha-\alpha_{1}-1}\right) \geq \underline{e}(t),
\end{gathered}
$$


and $\phi(t)$ and $\varphi(t) \in B$. Thus from (36)-(42), we have

$$
\begin{gathered}
D_{t}^{\alpha-\alpha_{1}} \varphi(t)+\lambda f\left(t, \mu \int_{0}^{1} K_{\beta}(t, s) g(s, \varphi(s)) d s\right) \\
=D_{t}^{\alpha-\alpha_{1}}(F \underline{e})(t)+\lambda f\left(t, \mu \int_{0}^{1} K_{\beta}(t, s) g(s,(F \underline{e})(s)) d s\right) \\
\leq D_{t}^{\alpha-\alpha_{1}}(F \underline{e})(t)+\lambda f\left(t, \mu \int_{0}^{1} K_{\beta}(t, s) g(s, \underline{e}(s)) d s\right)=0 \\
\varphi(0)=0, \quad D_{t}^{\gamma_{1}-\alpha_{1}} \varphi(1)=\sum_{j=1}^{m-2} b_{j} D_{t}^{\gamma_{1}-\alpha_{1}} \varphi\left(\zeta_{j}\right) \\
D_{t}^{\alpha-\alpha_{1}} \phi(t)+\lambda f\left(t, \mu \int_{0}^{1} K_{\beta}(t, s) g(s, \phi(s)) d s\right) \\
=D_{t}^{\alpha-\alpha_{1}}(F \bar{e})(t)+\lambda f\left(t, \mu \int_{0}^{1} K_{\beta}(t, s) g(s,(F \bar{e})(s)) d s\right) \\
\geq D_{t}^{\alpha-\alpha_{1}}(F \bar{e})(t)+\lambda f\left(t, \mu \int_{0}^{1} K_{\beta}(t, s) g(s, \bar{e}(s)) d s\right)=0 \\
\phi(0)=0, \quad D_{t}^{\gamma_{1}-\alpha_{1}} \phi(1)=\sum_{j=1}^{m-2} b_{j} D_{t}^{\gamma_{1}-\alpha_{1}} \phi\left(\zeta_{j}\right) .
\end{gathered}
$$

So it follows from (42)-(43) that $\varphi(t)$ and $\phi(t)$ are, respectively, upper solution and lower solution of the problem (28) and $\varphi(t), \phi(t) \in B$.

Define a function $W:(0,1) \times E \rightarrow E$ as follows:

$W(t, x(t))$

$$
= \begin{cases}f\left(t, \mu \int_{0}^{1} K_{\beta}(t, s) g(s, \phi(s)) d s\right), & x(t)<\phi(t), \\ f\left(t, \mu \int_{0}^{1} K_{\beta}(t, s) g(s, x(s)) d s\right), & \phi(t) \leq x(t) \leq \varphi(t), \\ f\left(t, \mu \int_{0}^{1} K_{\beta}(t, s) g(s, \varphi(s)) d s\right), & x(t)>\varphi(t) .\end{cases}
$$

Obviously $W \in C((0,1) \times[0,+\infty))$.

Next we define an operator $T$ in $C[0,1]$ by

$$
(T x)(t)=\lambda \int_{0}^{1} K_{\alpha}(t, s) W(s, x(s)) d s
$$

and consider the following boundary value problem:

$$
\begin{gathered}
-D_{t}^{\alpha-\alpha_{1}} x(t)=\lambda W(t, x(t)), \\
x(0)=0, \quad D_{t}^{\gamma_{1}-\alpha_{1}} x(1)=\sum_{j=1}^{m-2} b_{j} D_{t}^{\gamma_{1}-\alpha_{1}} x\left(\zeta_{j}\right) .
\end{gathered}
$$

Note that

$$
\begin{aligned}
& (T x)(t) \leq \lambda t^{\alpha-\alpha_{1}-1} \int_{0}^{1} M_{\alpha}(s) W(s, x(s)) d s \\
& \leq \lambda t^{\alpha-\alpha_{1}-1} \int_{0}^{1} M_{\alpha}(s) \\
& \quad \times f\left(s, \mu \int_{0}^{1} K_{\beta}(s, \tau) g(\tau, \phi(\tau)) d \tau\right) d s \\
& \leq \lambda t^{\alpha-\alpha_{1}-1} \int_{0}^{1} M_{\alpha}(s) \\
& \quad \times f\left(s, \mu \int_{0}^{1} K_{\beta}(s, \tau) g\left(\tau, l_{\phi} \tau^{\alpha-\alpha_{1}-1}\right) d \tau\right) d s
\end{aligned}
$$$$
<+\infty \text {; }
$$

then $T$ is uniformly bounded. From the uniform continuity of $K_{\alpha}(t, s)$ and Lebesgue dominated convergence theorem, we get $T$ is equicontinuous. So $T$ is completely continuous. It follows from Schauder's fixed point theorem that $T$ has at least one fixed point $x^{*}(t)$ such that $x^{*}(t)=\left(T x^{*}\right)(t)$.

In what follows, we prove

$$
\phi(t) \leq x^{*}(t) \leq \varphi(t), \quad t \in[0,1] .
$$

In fact, as $x^{*}(t)$ is a fixed point of $T$, we have

$$
x^{*}(0)=0, \quad D_{t}^{\gamma_{1}-\alpha_{1}} x^{*}(t)=\sum_{j=1}^{m-2} b_{j} D_{t}^{\gamma_{1}-\alpha_{1}} x^{*}\left(\zeta_{j}\right) .
$$

We firstly prove $x^{*}(t) \leq \varphi(t)$. Otherwise, suppose $x^{*}(t)>$ $\varphi(t)$. According to the definition of $W$, we have

$$
\begin{aligned}
-D_{t}^{\alpha-\alpha_{1}} x^{*}(t) & =\lambda W\left(t, x^{*}(t)\right) \\
& =\lambda f\left(t, \mu \int_{0}^{1} K_{\beta}(t, s) g(s, \varphi(s)) d s\right) .
\end{aligned}
$$

On the other hand, as $\varphi$ is an upper solution of (28), we have

$$
-D_{t}^{\alpha-\alpha_{1}} \varphi(t) \geq \lambda f\left(t, \mu \int_{0}^{1} K_{\beta}(t, s) g(s, \varphi(s)) d s\right) .
$$

Letting $z(t)=\varphi(t)-x^{*}(t)$, (50)-(51) imply that

$$
\begin{gathered}
D_{t}^{\alpha-\alpha_{1}} z(t)=D_{t}^{\alpha-\alpha_{1}} \varphi(t)-D_{t}^{\alpha-\alpha_{1}} x^{*}(t) \leq 0, \\
z(0)=0, \quad D_{t}^{\gamma_{1}-\alpha_{1}} z(t)=\sum_{j=1}^{m-2} b_{j} D_{t}^{\gamma_{1}-\alpha_{1}} z\left(\zeta_{j}\right) .
\end{gathered}
$$

It follows from Lemma 4 that

$$
z(t) \geq 0
$$

that is, $x^{*}(t) \leq \varphi(t)$ on $[0,1]$, which contradicts $x^{*}(t)>\varphi(t)$. Thus $\varphi(t) \geq x^{*}(t), t \in(0,1)$. In the same way, $x^{*}(t) \geq \phi(t)$. Consequently,

$$
\phi(t) \leq x^{*}(t) \leq \varphi(t)
$$

and then $x^{*}(t)$ is a positive solution of the problem (28). 
It follows from $\phi(t), \varphi(t) \in B$ that there exist four numbers $0<l_{\phi}, l_{\varphi}<1, L_{\phi}, L_{\varphi}>1$ such that

$$
\begin{aligned}
& l_{\phi} t^{\alpha-\alpha_{1}-1} \leq \phi(t) \leq L_{\phi} t^{\alpha-\alpha_{1}-1}, \\
& l_{\varphi} t^{\alpha-\alpha_{1}-1} \leq \varphi(t) \leq L_{\varphi} t^{\alpha-\alpha_{1}-1}
\end{aligned}
$$

and (54)-(55) yield

$$
r_{1} t^{\alpha-\alpha_{1}-1}=l_{\phi} t^{\alpha-\alpha_{1}-1} \leq x^{*}(t) \leq L_{\varphi} t^{\alpha-\alpha_{1}-1}=r_{2} t^{\alpha-\alpha_{1}-1} .
$$

Furthermore,

$$
\begin{aligned}
& d_{1} t^{\beta-\beta_{1}-1} \\
& \quad \leq \mu t^{\beta-\beta_{1}-1} \int_{0}^{1} m_{\beta}(s) g\left(s, r_{1} s^{\alpha-\alpha_{1}-1}(s)\right) d s \leq y^{*}(t) \\
& \leq \mu t^{\beta-\beta_{1}-1} \int_{0}^{1} M_{\beta}(s) g\left(s, r_{2} s^{\alpha-\alpha_{1}-1}(s)\right) d s \leq d_{2} t^{\beta-\beta_{1}-1}
\end{aligned}
$$

where

$$
\begin{aligned}
& d_{1}=\min \left\{\frac{1}{2}, \mu \int_{0}^{1} m_{\beta}(s) g\left(s, r_{1} s^{\alpha-\alpha_{1}-1}(s)\right) d s\right\}, \\
& d_{2}=\max \left\{2, \mu \int_{0}^{1} M_{\beta}(s) g\left(s, r_{2} s^{\alpha-\alpha_{1}-1}(s)\right) d s\right\} .
\end{aligned}
$$

By Lemma 1, (56), and (57), we obtain that the problem (3) has a positive solution $\left(u^{*}(t), v^{*}(t)\right)=\left(I^{\alpha_{1}} x^{*}(t), I^{\beta_{1}} y^{*}(t)\right)$, which satisfies

$$
\begin{aligned}
r_{1}^{*} t^{\alpha-1} & =\frac{\Gamma\left(\alpha-\alpha_{1}\right) r_{1}}{\Gamma(\alpha)} t^{\alpha-1} \leq u^{*}(t) \\
& =\frac{1}{\Gamma\left(\alpha_{1}\right)} \int_{0}^{t}(t-s)^{\alpha_{1}-1} x^{*}(s) d s \\
& \leq \frac{\Gamma\left(\alpha-\alpha_{1}\right) r_{2}}{\Gamma(\alpha)} t^{\alpha-1}=r_{2}^{*} t^{\alpha-1}, \\
d_{1}^{*} t^{\beta-1} & =\frac{\Gamma\left(\beta-\beta_{1}\right) d_{1}}{\Gamma(\beta)} t^{\beta-1} \leq v^{*}(t) \\
& =\frac{1}{\Gamma\left(\beta_{1}\right)} \int_{0}^{t}(t-s)^{\beta_{1}-1} y^{*}(s) d s \\
& \leq \frac{\Gamma\left(\beta-\beta_{1}\right) d_{2}}{\Gamma(\beta)} t^{\beta-1}=d_{2}^{*} t^{\beta-1} .
\end{aligned}
$$

The proof is completed.
Example 8. Consider the following singular fractional order differential system

$$
\begin{gathered}
-D_{t}^{7 / 4} u(t)=\lambda\left[\sin t+\left(D_{t}^{1 / 3} v(t)\right)^{-1 / 3}\right], \\
-D_{t}^{5 / 3} v(t)=\mu\left[t^{-1 / 2}+\left(D_{t}^{1 / 2} u(t)\right)^{2}\right], \\
D_{t}^{1 / 2} u(0)=0, \quad D_{t}^{2 / 3} u(1)=\sum_{j=1}^{10} b_{j} D_{t}^{2 / 3} u\left(\zeta_{j}\right), \\
D_{t}^{1 / 3} v(0)=0, \quad D_{t}^{3 / 5} v(1)=\sum_{j=1}^{10} a_{j} D_{t}^{3 / 5} v\left(\xi_{j}\right),
\end{gathered}
$$

where $\lambda>0$ and $\mu>0$ are parameters, $\xi_{j}=j / 12, a_{j}=$ $1 / 15 j^{1 / 15}, \zeta_{j}=1 /(j+1)$, and $b_{j}=(1 / 2)^{j}, j=1,2, \ldots, 10$. Obviously,

$$
\begin{aligned}
& 0<\sum_{j=1}^{10} a_{j} \xi_{j}^{1 / 15}=\sum_{j=1}^{10} \frac{1}{15 j^{1 / 15}}\left(\frac{j}{12}\right)^{1 / 15}=\frac{1}{15} \sum_{j=1}^{10} \frac{1}{12^{1 / 15}}<1, \\
& 0<\sum_{j=1}^{10} b_{j} \zeta_{j}^{1 / 12}=\sum_{j=1}^{10}\left(\frac{1}{2}\right)^{j}\left(\frac{1}{j+1}\right)^{1 / 12} \leq\left(\frac{1}{2}\right)^{1 / 13}<1 .
\end{aligned}
$$

Letting $u(t)=I^{1 / 2} x(t), v(t)=I^{1 / 3} y(t)$, then (60) is turned into

$$
\begin{gathered}
-D_{t}^{5 / 4} x(t)=\lambda\left(\sin t+y^{-1 / 3}(t)\right), \\
-D_{t}^{4 / 3} y(t)=\mu\left(t^{-1 / 2}+x^{2}(t)\right), \\
x(0)=0, \quad D_{t}^{1 / 3} x(1)=\sum_{j=1}^{10} b_{j} D_{t}^{1 / 3} x\left(\zeta_{j}\right), \\
y(0)=0, \quad D_{t}^{1 / 10} y(1)=\sum_{j=1}^{10} a_{j} D_{t}^{1 / 10} y\left(\xi_{j}\right),
\end{gathered}
$$

where $f(t, y)=\sin t+y^{-1 / 3}$ and $g(t, x)=t^{-1 / 2}+x^{2}$; it is easy to see that $f(t, y)$ is decreasing with respect to $y$ and $g(t, x)$ is increasing with respect to $x$, and then (A1) is satisfied.

It follows from (20) and (21) that there exists a positive number $m_{0}<+\infty$ such that

$$
K_{\beta}(t, s) \geq m_{\beta}(s) t^{1 / 3} \geq m_{0} t^{1 / 3},
$$

and for any constants $\mu, l>0$, we have

$$
\begin{aligned}
& f\left(t, \mu \int_{0}^{1} K_{\beta}(t, s) g\left(s, l s^{\alpha-\alpha_{1}-1}\right) d s\right) \\
& =\sin t+\left(\mu \int_{0}^{1} K_{\beta}(t, s) g\left(s, l s^{\alpha-\alpha_{1}-1}\right) d s\right)^{-1 / 3} \\
& =\sin t+\left(\mu \int_{0}^{1} K_{\beta}(t, s)\left(s^{-1 / 2}+l^{2} s^{2}\right) d s\right)^{-1 / 3}
\end{aligned}
$$




$$
\begin{aligned}
& \leq \sin t+\left(\mu m_{0}\right)^{-1 / 3}\left(\int_{0}^{1} s^{-1 / 2}+l^{2} s^{2} d s\right)^{-1 / 3} \\
\leq & \sin 1+\left(\mu m_{0}\right)^{-1 / 3}\left(\frac{1}{2}+\frac{1}{3} l^{2}\right)^{-1 / 3} \\
& <\sin 1+\left(\frac{1}{2} \mu m_{0}\right)^{-1 / 3}, \\
M_{\alpha}(s)= & \frac{1}{\Gamma(5 / 4)}+\frac{\sum_{j=1}^{10} b_{j} K_{\alpha 2}\left(\zeta_{j}, s\right)}{1-c_{2}} \leq \frac{1}{\Gamma(5 / 4)} \\
& +\frac{\left((1-s)^{1 / 12} / \Gamma(5 / 4)\right) \sum_{j=1}^{10}(j+1)(1 / 2)^{j}}{1-c_{2}} \\
\leq & \frac{1}{\Gamma(5 / 4)}\left(1+\frac{2^{10}-9}{2^{11}\left(1-c_{2}\right)}\right)
\end{aligned}
$$

where $c_{2}=\sum_{j=1}^{10} b_{j} \zeta_{j}^{1 / 12}$. So

$$
\begin{aligned}
& \int_{0}^{1} M_{\alpha}(s) f\left(s, \mu \int_{0}^{1} K_{\beta}(s, \tau) g\left(\tau, l \tau^{\alpha-\alpha_{1}-1}\right) d \tau\right) d s \\
& \leq \frac{1}{\Gamma(5 / 4)} \int_{0}^{1}\left(\sin 1+\left(\frac{1}{2} \mu m_{0}\right)^{-1 / 3}\right)\left(1+\frac{2^{10}-9}{2^{11}\left(1-c_{2}\right)}\right) d s \\
& <+\infty,
\end{aligned}
$$

and thus (A2) is satisfied. So from Theorem 7, for any $(\lambda, \mu) \epsilon$ $(0,+\infty) \times(0,+\infty)$, the system $(60)$ has at least one positive solution $\left(u^{*}, v^{*}\right)$, and there exist positive constants $r_{1}^{*}, r_{2}^{*}, d_{1}^{*}$, and $d_{2}^{*}$ such that

$$
\begin{array}{r}
r_{1}^{*} t^{3 / 4} \leq u^{*}(t) \leq r_{2}^{*} t^{3 / 4}, \quad d_{1}^{*} t^{2 / 3} \leq v^{*}(t) \leq d_{2}^{*} t^{2 / 3}, \\
t \in[0,1] .
\end{array}
$$

\section{Conflict of Interests}

The authors declare that there is no conflict of interests regarding the publication of this paper.

\section{Acknowledgments}

The authors were supported financially by the National Natural Science Foundation of China (no. 11371221) and the Project of Shandong Province Higher Educational Science and Technology Program (no. J13LI12).

\section{References}

[1] A. A. Kilbas, H. M. Srivastava, and J. J. Trujillo, "Theory and applications of fractional differential equations," in NorthHolland Mathematics Studies, vol. 204, Elservier Science, Amsterdam, The Netherlands, 2006.

[2] I. Podlubny, Fractional Differential Equations, Academic Press, San Diego, Calif, USA, 1999.
[3] J. Sabatier, O. P. Agrawal, and J. A. T. Machado, Eds., Advances in Fractional Calculus: Theoretical Developments and Applications in Physics and Engineering,, Springer, Dordrecht, The Netherlands, 2007.

[4] S. G. Samko, A. A. Kilbas, and O. I. Marichev, Fractional Integral and Derivatives, Theory and Applications, Gordon and Breach Science, Yverdon, Switzerland, 1993.

[5] A. S. Perelson, "Modeling the interaction of the immune system with HIV," in Mathematical and Statistical Approaches To AIDS Epidemiology, C. Castillo-Chavez, Ed., vol. 83 of Lecture Notes in Biomathematics, Springer, New York, NY, USA, 1989.

[6] C. S. Goodrich, "Existence of a positive solution to systems of differential equations of fractional order," Computers \& Mathematics with Applications, vol. 62, no. 3, pp. 1251-1268, 2011.

[7] X. Zhang, L. Liu, and Y. Wu, "Multiple positive solutions of a singular fractional differential equation with negatively perturbed term," Mathematical and Computer Modelling, vol. 55, no. 3-4, pp. 1263-1274, 2012.

[8] B. Ahmad and J. Nieto, "Existence results for a coupled system of nonlinear fractional differential equations with three-point boundary conditions," Computers \& Mathematics with Applications, vol. 58, no. 9, pp. 1838-1843, 2009.

[9] C. S. Goodrich, "Existence and uniqueness of solutions to a fractional difference equation with nonlocal conditions," Computers and Mathematics with Applications, vol. 61, no. 2, pp. 191-202, 2011.

[10] C. S. Goodrich, "Positive solutions to boundary value problems with nonlinear boundary conditions," Nonlinear Analysis, Theory, Methods and Applications, vol. 75, no. 1, pp. 417-432, 2012.

[11] A. A. M. Arafa, S. Z. Rida, and M. Khalil, "Fractional modeling dynamics of HIV and $\mathrm{CD}^{+}$T-cells during primary infection," Nonlinear Biomedical Physics, vol. 6, no. 1, article 1, 2012.

[12] X. Zhang, L. Liu, and Y. Wu, "The uniqueness of positive solution for a singular fractional differential system involving derivatives," Communications in Nonlinear Science and Numerical Simulation, vol. 18, no. 6, pp. 1400-1409, 2013.

[13] X. Zhang, L. Liu, and Y. Wu, "The eigenvalue problem for a singular higher order fractional differential equation involving fractional derivatives," Applied Mathematics and Computation, vol. 218, no. 17, pp. 8526-8536, 2012.

[14] X. Zhang, L. Liu, and Y. Wu, "Existence results for multiple positive solutions of nonlinear higher order perturbed fractional differential equations with derivatives," Applied Mathematics and Computation, vol. 219, no. 4, pp. 1420-1433, 2012.

[15] I. S. Jesus, J. A. Tenreiro MacHado, and J. Boaventure Cunha, "Fractional electrical impedances in botanical elements," JVC/Journal of Vibration and Control, vol. 14, no. 9-10, pp. 1389-1402, 2008.

[16] X. Zhang, L. Liu, B. Wiwatanapataphee, and Y. Wu, "Positive solutions of eigenvalue problems for a class of fractional differential equations with derivatives," Abstract and Applied Analysis, vol. 2012, Article ID 512127, 16 pages, 2012.

[17] M. Rehman and R. Khan, "Existence and uniqueness of solutions for multi-point boundary value problems for fractional differential equations," Applied Mathematics Letters, vol. 23, pp. 1038-1044, 2010

[18] X. Zhang, L. Liu, Y. Wu, and Y. Lu, "The iterative solutions of nonlinear fractional differential equations," Applied Mathematics and Computation, vol. 219, no. 9, pp. 4680-4691, 2013. 


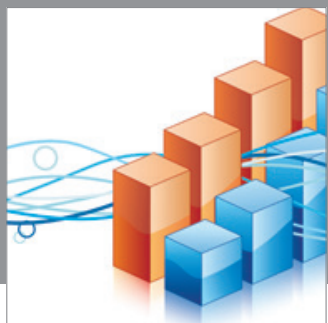

Advances in

Operations Research

mansans

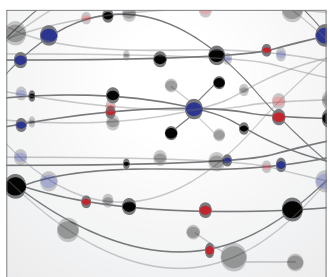

The Scientific World Journal
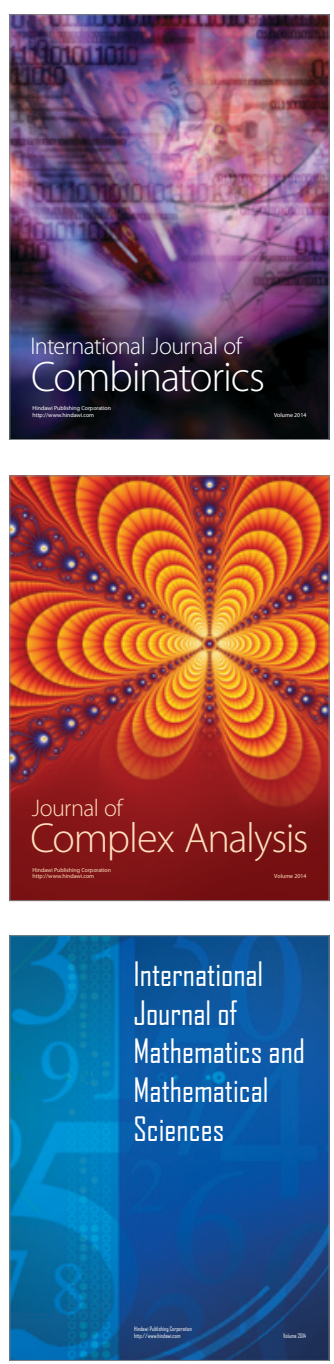
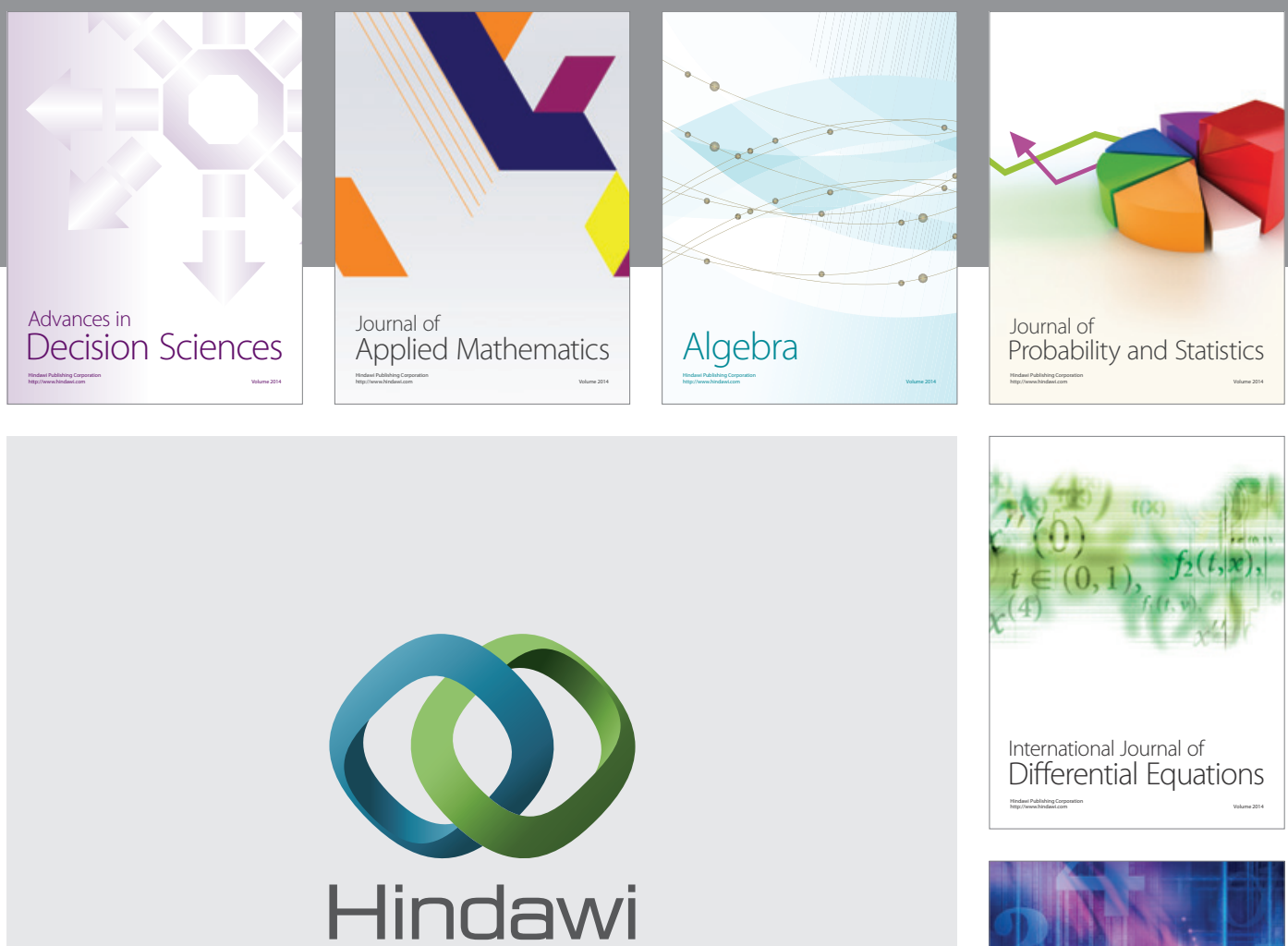

Submit your manuscripts at http://www.hindawi.com
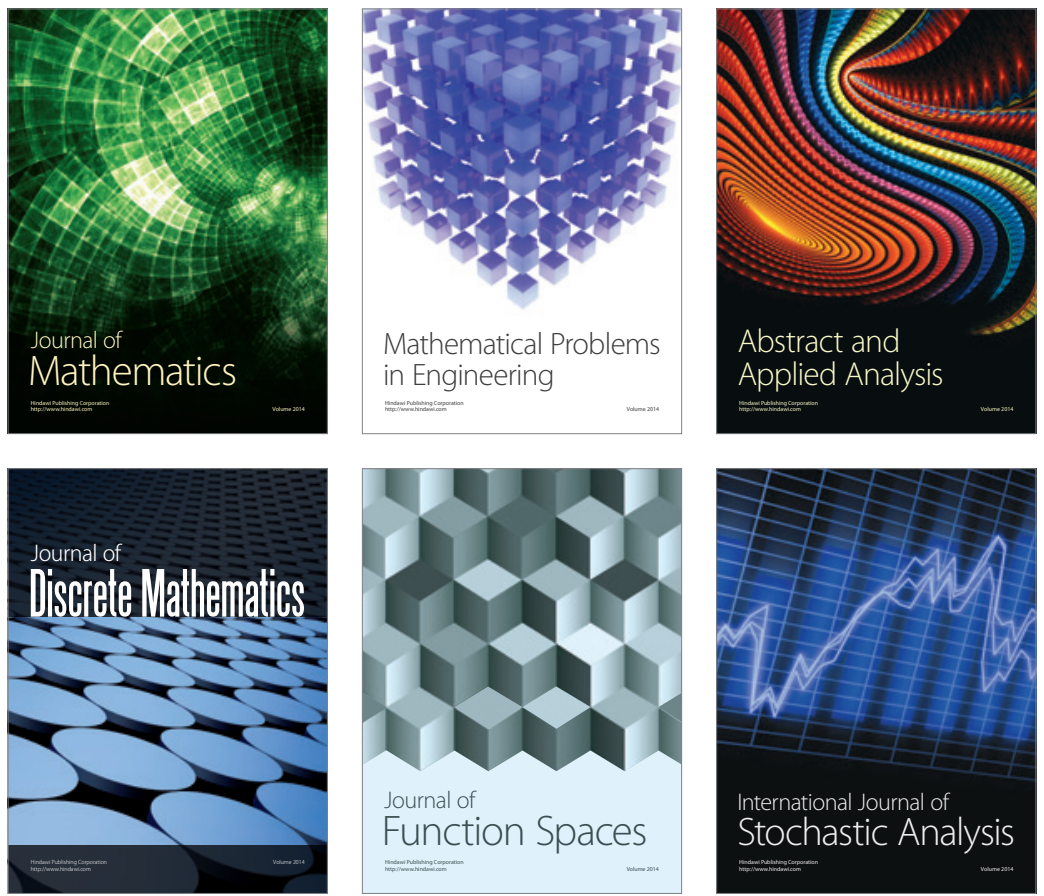

Journal of

Function Spaces

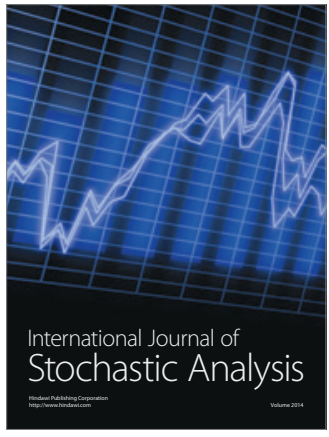

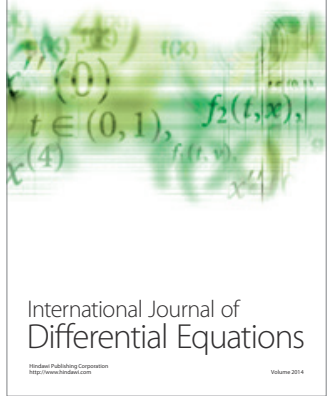
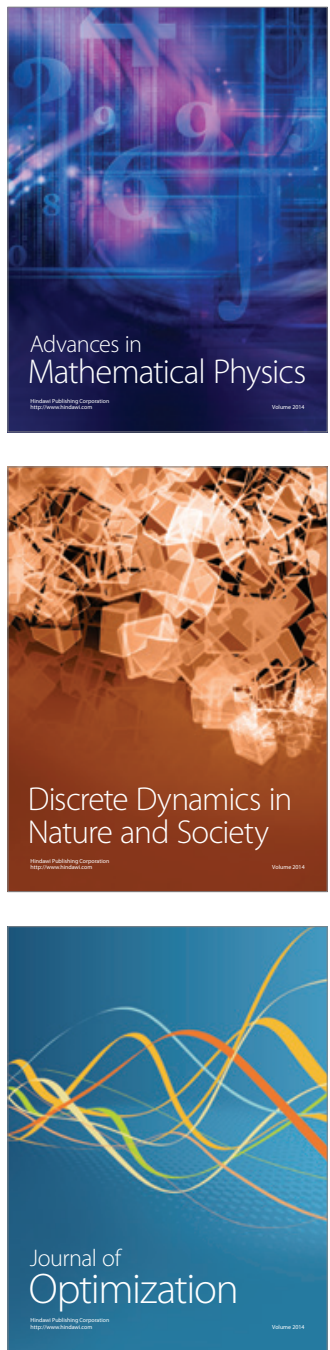